\title{
Taxonomía de la diplomacia digital en la agenda de las nuevas relaciones internacionales
}

\author{
Juan Luis MANFREDI SÁNCHEZ \\ Universidad de Castilla-La Mancha \\ Juan.manfredi@uclm.es
}

Recibido: 8 de julio de 2014

Aceptado: 10 de octubre de 2014

\begin{abstract}
Resumen
La diplomacia digital se ha convertido en uno de los elementos fundamentales para comprender la transformación de la diplomacia contemporánea. El análisis de los principios, métodos y fines demuestra que estamos aún en una fase muy inicial de transformación digital. El artículo analiza y compara las principales actuaciones de Estados Unidos, Francia, Suecia y el Servicio Europeo de Acción Exterior.
\end{abstract}

Palabras clave: Diplomacia; comunicación internacional; diplomacia digital; transparencia.

\section{Taxonomy of digital diplomacy in the international affairs agenda}

\begin{abstract}
Digital diplomacy has become one of the key elements for understanding the transformation of contemporary diplomacy. The analysis of the principles, methods and purposes shows that we are still in a very early stage of digital transformation.
\end{abstract}

Key words: Diplomacy; international communication; digital diplomacy; transparency.

\section{Referencia normalizada}

Manfredi Sánchez, J. L. (2014) Taxonomía de la diplomacia digital en la agenda de las nuevas relaciones internacionales. Historia y Comunicación Social. Vol. 19. Páginas 341-354.

Sumario: 1. Introducción: la transformación de la diplomacia contemporánea. 2. Metodología. 3. Estado de la cuestión. 4. Taxonomía de la diplomacia digital. 5. Conclusiones. 6. Referencias bibliográficas. 


\section{Introducción: La transformación de la diplomacia contemporánea}

La diplomacia contemporánea se enfrenta a un momento de cambio ante la emergencia de nuevos actores que tienen capacidad para influir en la agenda de las relaciones internacionales (Manfredi, 2011). Tras la caída del muro, los autores buscaron nuevas formulaciones teóricas sobre el poder en relaciones internacionales. Manuel Castells plantea que la relevancia de la comunicación estratégica, la producción social del significado y la batalla por la opinión pública ha crecido en la sociedad red: "las relaciones de poder, es decir, las relaciones que constituyen los fundamentos de toda sociedad, además de los procesos que desafían las relaciones de poder institucionalizadas, se determinan y deciden cada vez más en el campo de la comunicación" (2008:13). Anne-Marie Slaughter teoriza sobre el valor de las redes y las conexiones como fuentes de poder, hasta el punto de que "el poder que fluye de este tipo de conexiones no es la capacidad de imponer objetivos. Las redes no son dirigidas y controladas sino que son gestionadas y orquestadas. Los múltiples actores se integran en un todo que es más relevante que la suma de las partes - una orquesta que toca de diferentes maneras de acuerdo con la visión de su director y el talento individual de los músicos" (2009:99-100). En la misma línea, Joseph Nye ha reformulado la idea del poder blando hasta convertirlo en el poder inteligente, una suerte de "hábil combinación de poder blando y poder duro. Significa el desarrollo de una estrategia integrada, dotada de recursos y herramientas para conseguir los objetivos de Estados Unidos", en la terminología de Armitage y Nye (2007:7). Entre los nuevos objetivos que se plantean en la acción exterior de los estados, se consolida la comunicación internacional. La creciente preocupación por la imagen exterior del país se concreta en las oficinas de marca país, las estrategias de diplomacia pública o las campañas internacionales de captación de inversiones, iniciativa ésta que mezcla la acción pública con la privada. No es novedoso que el diplomático se ocupe de la imagen de su país; la novedad consiste en que ahora lo hace de forma profesional y sistemática, incluida en su lista de tareas.

Los acuerdos internacionales y los encuentros entre representantes son de naturaleza privada, pero sus resultados y consecuencias son públicas y previsibles. Tras el final de la Guerra Fría, la dimensión militar y secreta de la diplomacia ha declinado. Los encuentros, desde las Cumbres Iberoamericanas hasta los Acuerdos de Ginebra II, se ven, se agenda y se comunican. Los acuerdos no normativos, las declaraciones y los comunicados conjuntos han alimentado el sistema de consultas que facilita las relaciones diplomáticas (Estrella, 2011).

El enfoque holístico de las relaciones internacionales sugiere la idea de una diplomacia en red. Hocking (2006:13) señala que "la diplomacia se ha convertido en una actividad que consiste en la creación de redes, abrazando una amplia gama de estados y actores no estatales, que se centran en la gestión de asuntos que demandan recursos sobre los cuales ningún participante por sí solo posee el monopolio". Gstöl (2012:57) habla del nuevo mapa de competencias que el profesional de la diplomacia debe adquirir: "el enfoque de redes sugiere que el diplomático contemporáneo debe ser 
un generalista que ha adquirido el conocimiento y las habilidades que hacen de él o ella un "especialista en el arte de la diplomacia", un maestro en la gestión de las relaciones y la complejidad o, de manera más simple, un excelente creador de redes". Jorge Heine (2008) describe el cambio como la transformación de un modelo de diplomacia club, compuesta por diplomáticos y otros altos cargos oficiales, hacia un modelo de diplomacia red, que obliga a los diplomáticos a incrementar y mantener relaciones con otros actores que carecen de esa oficialidad.

La multiplicidad de fuentes de legitimación, participación y comunicación apuntan esa hipótesis de una diplomacia en red, en la que las actividades digitales ocupan un espacio creciente. Internet y los nuevos medios suponen una nueva transformación de la actividad diplomática, cuyas funciones centrales (representar, negociar, proteger y promover los intereses de un estado ante terceros) se han visto volteadas por la comunicación internacional, los medios digitales y diplomacia en tiempo real. Han afectado a la agenda pública, al activismo y a la participación política en la arena internacional. Ya se habla con soltura de la diplomacia digital en medios académicos y profesionales.

El propio Joseph Nye (2013) habla de una diplomacia 3.0, que abraza la sociedad de la información y reconoce el poder de los ciudadanos para comunicarse, relacionarse y establecer conexiones. Las redes sociales y la influencia digital extienden la idea de poder hacia nuevos ámbitos de relación. Phil Seib prefiere hablar de una diplomacia en tiempo real, que actúa en un entorno mediático que requiere gran velocidad de respuesta y transparencia. Sostiene Seib que "la era en la que los diplomáticos hablaban solo con otros diplomáticos y programaban sus conversiones a su gusto ha pasado. Estamos ante un nuevo tiempo, las nuevas prácticas diplomáticas tiene que desempeñarse para triunfar en la era del tiempo real" (2012:86). Esta transformación de la actividad diplomática tiene consecuencias en los programas de diplomacia pública, que ha sido la primera actividad revisada.

\section{Metodología}

El presente trabajo es un análisis descriptivo de los modelos de diplomacia contemporánea, con especial atención a la dimensión digital de la actividad. Se repasa la nueva bibliografía que actualiza el estudio de las relaciones entre los estados, así como la emergencia de nuevos actores que reclaman su espacio en la arena internacional. Las nuevas ideas que configuran la diplomacia en red requieren una redefinición y una contextualización de qué riesgos, oportunidades y acciones pueden emprenderse en este entorno. Finalmente, el texto muestra las principales aportaciones prácticas ejecutadas por los servicios de acción exterior de Estados Unidos, Suecia, Francia y el Servicio Europeo de Acción Exterior. La novedad del trabajo es el estudio del fenómeno digital, las implicaciones y la relevancia efectiva. Partimos de un hecho concreto: la inclusión de las tecnologías en la actividad diplomática 
significa la ampliación del rango de actividades, actores implicados y oportunidades para la gestión.

El artículo presenta una estructura compuesta por los siguientes elementos. El primero repasa los principales autores en materia de sociedad red, con atención a las transformaciones del estado y sus relaciones internacionales. Incluimos la idea de poder inteligente y diplomacia 3.0, comúnmente aceptados en la literatura. Seguidamente, analizamos cómo la comunicación internacional se ha insertado en la actividad diplomática y cómo el entorno digital ha acelerado la dimensión pública de la diplomacia. Concluimos con un estudio de las acciones concretas que ya se han ejecutado para comprobar el grado de novedad en la práctica y planteamos cuáles serán los retos de la investigación en la relación entre comunicación y diplomacia a medio plazo.

\section{Estado de la cuestión}

La diplomacia consiste en el arte de representar, negociar, proteger y promover los intereses de un Estado ante terceros, según recoge el Convenio de Viena sobre Relaciones Diplomáticas (1961). Es una actividad profesional cuya dimensión comunicativa es intensa. Dos de las funciones de la misión diplomática, de acuerdo con dicho convenio, se vinculan con la comunicación. Por un lado, el diplomático ha de "enterarse por todos los medios lícitos de las condiciones y de la evolución de los acontecimientos en el Estado receptor e informar sobre ello al gobierno del Estado acreditante" (artículo 3.d). Por otro, se persigue "fomentar las relaciones amistosas y desarrollar las relaciones económicas, culturales y científicas entre el Estado acreditante y el Estado receptor" (artículo 3.e). La perspectiva cultural y comunicativa está en la esencia de las relaciones entre estados. Arndt indica que "cualquier diplomacia significa(ba) el establecimiento de relaciones no entre naciones-estado, sino entre culturas" (2005:1).

En el articulado, la comunicación convencional reaparece como un derecho limitado. El artículo 27 recoge la garantía de la libre comunicación de la misión con su gobierno, la inviolabilidad de la correspondencia y la valija. No obstante, limita expresamente la instalación y utilización de una emisora de radio. Por extensión, se entiende que las misiones diplomáticas pueden emplear los medios de comunicación al uso pero no pueden crearlos ni producirlos.

Estas premisas tan genéricas confirman que la comunicación es una actividad preferente de la diplomacia, cuya dimensión pública ha crecido por la concatenación de tres factores concatenados (Seib,2012). El primero es la consolidación de los medios audiovisuales internacionales. El estudio del efecto CNN (Livingston, 1997) está obsoleto. Hoy día sería muy complejo examinar los efectos mediáticos de una intervención militar televisada en la medida que $\mathrm{CNN}$, ni ninguna otra cadena, monopoliza los mensajes en el sistema periodístico. Al menos ocho grandes cadenas 
internacionales compiten en el mercado audiovisual (tabla 1). Con diferente titularidad, financiación y misión, han conseguido normalizar las noticias internacionales, dispersar las audiencias y promover contenidos periodísticos con diferentes framings (Azpiroz, 2013).

Las ocho grandes cadenas internacionales (datos propios y públicos según sus respectivas web corporativas, 2014)

Tabla 1

\begin{tabular}{|l|l|l|l|}
\hline Cadena & $\begin{array}{l}\text { Nacionalidad de } \\
\text { referencia }\end{array}$ & $\begin{array}{l}\text { Titularidad / } \\
\text { Financiación }\end{array}$ & Misión \\
\hline RT (2005) & Rusia & Pública/Mixta & $\begin{array}{l}\text { Contar al mundo historias sobre } \\
\text { Rusia e informar sobre las noticias } \\
\text { internacionales desde una perspec- } \\
\text { tiva rusa }\end{array}$ \\
\hline CCTV & China & Pública/Pública & $\begin{array}{l}\text { Dar voz a la perspectiva china de } \\
\text { los asuntos internacionales y rom- } \\
\text { per el monopolio de las voces occi- } \\
\text { dentales de las noticias }\end{array}$ \\
\hline Deutsche Welle DW & Alemania & Pública/Pública & $\begin{array}{l}\text { Dar forma a la innovación. Explore } \\
\text { el mundo con reconocidos expertos }\end{array}$ \\
\hline Al Jazeera & Qatar & Pública/Privada & $\begin{array}{l}\text { Noticias independientes e imparcia- } \\
\text { les para una audiencia internacio- } \\
\text { nal. Ofrecer una voz para contribuir } \\
\text { a la diversidad de perspectivas de } \\
\text { los países menos representados. } \\
\text { Aspira a convertirse en la voz de } \\
\text { "los sin voz" }\end{array}$ \\
\hline $\begin{array}{l}\text { Radio France Inter- } \\
\text { nationale }\end{array}$ & Francia & Pública/ Pública & $\begin{array}{l}\text { La cobertura de los acontecimientos } \\
\text { desde una perspectiva francesa. } \\
\text { La promoción de los valores fran- } \\
\text { ceses a través del mundo. }\end{array}$ \\
\hline $\begin{array}{l}\text { BBG Broadcasting } \\
\text { Board of Governors }\end{array}$ & Estados Unidos & Pública/Mixta & $\begin{array}{l}\text { Informar, interactuar y conectar con } \\
\text { personas alrededor del mundo para } \\
\text { la promoción de la libertad y la } \\
\text { democracia }\end{array}$ \\
\hline BBC - Global News & Reino Unido & Pública/Pública & $\begin{array}{l}\text { Primeros en las noticias de actua- } \\
\text { lidad. } \\
\text { Más formas innovadoras cada vez }\end{array}$ \\
\hline Estados Unidos & $\begin{array}{l}\text { La provisión de noticias internacio- } \\
\text { nales confiables } \\
\text { Dotar de información y análisis de } \\
\text { la mejor calidad }\end{array}$ \\
\hline
\end{tabular}

Mención especial merece Al Jazeera, cuya audiencia e influencia ha crecido de forma exponencial. Según datos de la propia cadena, emite en 100 países y está en 220 millones de hogares. En el ámbito de las redes sociales, la cadena qatarí ha sabido aprovechar la transformación del ecosistema periodístico (Marshall, 2013). Algunas experiencias notables son la cobertura de la guerra de Gaza en 2008 y 2009. En aquel 
momento se crea la primera cuenta de Twitter (@AJGaza) con una finalidad periodística informativa. A finales de 2008, ya contaba con 17.000 seguidores. La experiencia conduce a cambiar el nombre a otro más global: @AJELive. La experiencia ha continuado en la cobertura de las elecciones en Irán, que plantea los primeros problemas de verificación de fuentes y contextualización. En 2010, se alía con Youtube para diseminar contenidos con ocasión de las elecciones iraquíes. El equipo periodístico de campo se componía de 17 profesionales dotados de cámaras digitales. Durante las revueltas del norte de África Al Jazeera se ha convertido en la televisión de referencia internacional. Ha aprovechado la experiencia acumulada para agregar, seleccionar y multiplicar los contenidos exclusivos elaborados por los propios televidentes, al tiempo que ha expandido su influencia a nuevos territorios.

El segundo elemento es la penetración de los medios digitales, que ha devenido esencial para comprender la transformación de la actividad diplomática es la consolidación del entorno digital como un espacio de acción política. La defensa de los intereses de los estados principia ahora en Internet, las redes sociales y los nuevos medios. La reputación, el prestigio y la influencia se refuerzan o debilitan en este entorno. El entorno digital compuesto por redes sociales, plataformas digitales y ciudadanos: son agregadores, mensajería instantánea, redes sociales generalistas y especializadas, fotografía, video, medios de comunicación y otras formulaciones mixtas. Los medios digitales impactan en la diplomacia en la medida que supera las fronteras administrativas y no infringe normas internacionales. Por eso, no se puede bloquear fácilmente la circulación de las ideas emitidas en el entorno digital ni cabe un censura sistemática, como podía suceder con los medios analógicos. Por su naturaleza, la dinámica y el alcance tiene una dimensión internacional lo que contribuye a la fragmentación de las audiencias. En suma, las redes vehiculan los mensajes, las ideologías, la producción cultural y las propuestas de renovación política. Ha empoderado a los ciudadanos, que unidos a través de estas herramientas, han promovido cambios con desigual éxito.

El tercer elemento de contexto es el auge de la transparencia, la filtración y la inteligencia. El fenómeno de Wikileaks es el epítome de este cambio de paradigma Beckett (2012) plantea que es un acontecimiento que desafía el periodismo convencional en la medida que afecta a la profesión, las fuentes, la clase política y las relaciones internacionales. El más reciente de la NSA refleja que el control y la fiscalización de la información sigue siendo un valor para las relaciones internacionales. Es el eterno debate que conjuga los elementos del periodismo, la alta política internacional, la libertad y la seguridad. Afecta las organizaciones políticas, pero también a la protección de los datos personales y la información confidencial.

En el ámbito periodístico, encontramos dos realidades contrapuestas. Por un lado, el periodismo de investigación ha aprendido a sacar provecho de las bases de datos, las fuentes públicas y otras fuentes abiertas. Destaca el trabajo de The International Consortium of Investigative Journalists (ICIJ), que elabora historias de impacto mundial centrados en la corrupción y la rendición de cuentas de los poderes establecidos. Cuenta con una red de 160 periodistas asentados en 60 países. Por otro, King 
(2014) sostiene que "las revelaciones sobre el espionaje han cambiado la forma en que los periodistas deben pensar sobre la seguridad de su trabajo, sobre sus fuentes y sobre ellos mismos. Los periodistas más prudentes que quisieran evitar el escrutinio de su trabajo o el de sus fuentes deberán adaptar sus comportamientos, bien evitando el contacto con las fuentes o bien empleando tecnologías que protegen la privacidad, como la encriptación. Este tipo de cambios impide a los periodistas reunir y diseminar información".

La ciudadanía ha tomado conciencia de la rendición de cuentas y es fuente de activismo digital. La literatura académica es extensa. No existe consenso sobre cuánto o cómo afectan los nuevos medios a la estructura política, pero no hay dudas sobre el impacto en Ucrania, Birmania, Túnez, Egipto, España, México o Brasil. La serie Internet \& Democracy Case Study Series, editada por Harvard University, ofrece un repertorio extenso.

Por su parte, los gobiernos han promovido leyes de transparencia con el ánimo de ampliar la rendición de cuentas (Guichot, 2012). Los sistemas democráticos están bajo control parlamentario, por lo que no cabe el ocultamiento de las posiciones en materia de acción exterior. No se concibe una actividad política secreta. El empoderamiento de la ciudadanía digital y de los movimientos sociales han contribuido a la construcción de una agenda pública abierta, renovadora de las fuentes de legitimidad de los sistemas y los procesos democráticos (Rubio, 2012).

Este contexto conduce al planteamiento del trilema de la diplomacia contemporánea. Un trilema es una situación incómoda para el análisis racional. Consiste en la elección entre tres alternativas que son incompatibles entre sí. No se obtiene una respuesta satisfactoria y equilibrada, por lo que es necesario elegir y establecer prioridades. En ciencia política, el trilema que plantea Dani Rodrik (2011) es el más conocido. Las tres alternativas son hiperglobalización, estado nación y democracia política. Las combinaciones que funcionan solo consiguen unir dos de los elementos, pero no los tres.

En esa línea conceptual, resulta complicado establecer una estrategia que combine los tres grandes dilemas en conflicto: la libertad y la seguridad; la transparencia y la confidencialidad; la libertad de expresión frente al discurso del odio. El trilema está permanentemente presente en la diplomacia digital.

\section{Taxonomía de la diplomacia digital}

Los principios de la diplomacia digital son la flexibilidad, la escalabilidad y la supervivencia, ejes de la sociedad red que plantea Manuel Castells (2009:23). La flexibilidad consiste en la capacidad de conseguir unos objetivos de política exterior mediante el uso de instrumentos no convencionales y la interconexión con otros participantes de la denominada diplomacia en red. La escalabilidad se manifiesta en la capacidad de crecer de forma exponencial sin incrementar los costes de produc- 
ción, esto es, los recursos destinados a la consecución de los objetivos de la política exterior. Por eso, la diplomacia red necesita la contribución y la cooperación de terceros. La supervivencia pasa por la habilidad para reconfigurarse, buscar aliados y encontrar puntos de encuentro.

Alec Ross, asesor de innovación de la secretaría de Estado de Estados Unidos entre 2009 y 2013, ha acuñado la expresión 21st Century Statecraft para indicar cómo esos principios transforman la actividad diplomática. Consiste en el desarrollo de instrumentos que complementen los propios de la política exterior. Es una apuesta por la innovación diplomática que combina redes, tecnologías y el cambio demográfico en un mundo de creciente interconexión. Incluye el reconocimiento del uso que los ciudadanos hacen de las redes sociales para la promoción de sus intereses, que pueden concordar o no con la política exterior del país en el que viven.

Los principios dan sentido a la propuesta programática de la diplomacia digital. Cada país los adopta de acuerdo con su acción exterior, su política exterior y sus instrumentos preferentes (diplomacia convencional, asistencia consular, inteligencia, cooperación al desarrollo o acción cultural, captación de inversiones e internacionalización, entre otros). En el caso del Ministerio de Asuntos Exteriores de Francia, los ejes de "diplomacia de influencia digital" son el refuerzo del diálogo con la sociedad civil francesa y extranjera; consolidar la dimensión de servicio público de France Diplomatie y más generalmente, la calidad del servicio ofrecido; respaldar el servicio de las representaciones diplomáticas en la comunidad digital (Diplomatie. gouv,2014). En el caso de Canadá, el propio ministro promueve el empleo de las tecnologías para la promoción de la democracia y las libertades, la defensa de los derechos humanos, así como la extensión del rule of law: "la diplomacia directa es un instrumento de la diplomacia contemporánea" (Baird, 2013).

Los fines de la diplomacia digital son la consecución de los objetivos de la política exterior de un país mediante el uso intensivo de la web social, las plataformas digitales y otras herramientas intensivas en tecnologías. La diplomacia digital no tiene una finalidad diferente de la convencional ni un fin por sí misma. En general, la Convención de Viene establece que la diplomacia tiene cuatro objetivos principales: representar, negociar, proteger y promover los intereses de un estado ante terceros. Como extensión de la diplomacia pública al uso, el ámbito digital requiere la actualización de los cinco componentes de la misma (Cull, 2008): la capacidad de escucha, la promoción de intereses, la diplomacia cultural, los intercambios y las emisiones internacionales de radio y televisión. La experiencia internacional es diversa. En el ámbito de la seguridad internacional, James Stavridis (2013) escribe que uno de las finalidades de la diplomacia digital es el establecimiento de un nuevo modelo de seguridad, abierto. Ante la multiplicación de elementos y actores de la diplomacia red, la seguridad necesita beneficiarse de los medios, las operaciones y la experiencia del entorno digital.

Así de esta manera, la virtud de la diplomacia digital reside no tanto en la creación de nuevos objetivos de política exterior, sino la capacidad de extender las redes de 
influencia. Francia reconoce que "la diplomacia de la influencia digital es la promoción de la imagen de Francia y, por ende, la defensa de sus intereses, ya sean económicos, lingüísticos o culturales" (Diplomatie.gouv,2014). Como vemos, son objetivos genéricos de Francia que ahora encuentran nuevas condiciones.

Hillary Clinton, en su conocido discurso "Internet Rights and Wrongs: Choices and Challenges in a Networked World" (2011), señala que Internet se ha convertido en parte fundamental del espacio público, por lo que la libertad de conexión ha emergido como una demanda sólida de la ciudadanía. Ésta consiste en la capacidad de participar, recibir y enviar información o exponer un punto de vista propio. Internet es la plaza pública en la que compiten las ideas, donde se intercambian experiencias y donde los ciudadanos viven parte de su vida política. Por eso, Internet se convierte en fundamento de la nueva esfera pública en el siglo XXI. En suma, se entiende la diplomacia digital como una vía para la promoción de los objetivos de la diplomacia convencional. No hay nada exclusivo o diferencial.

Los métodos de trabajo son el conjunto de herramientas que se emplean para la consecución de objetivos. Pueden ser a corto o largo plazo, dirigida a audiencias especializadas o generalistas, apostar por las tácticas o ser estratégicas. Cada objetivo requerirá unos instrumentos adecuados a la circunstancia concreta. La diplomacia digital amplia el abanico de herramientas y facilita el contacto con otros ciudadanos. Es diplomacia pública en la medida que el diplomático hace público su trabajo. Pero esta nueva aproximación requiere nuevas habilidades tanto para el diplomático como para las cancillerías.

La transformación digital en el área diplomática tiene consecuencias en las habilidades y las competencias profesionales. La crítica más extendida a los documentos de Wikileaks es la baja calidad de la información que allí se contiene. Están repletos de información, pero no de conocimiento. No hay nada en ellos que revele propósitos de la política exterior estadounidense que no sean conocidos de una u otra manera. La narrativa y los instrumentos parecen extraídos de otro tiempo. Apunta Seib: "algunos de los grandes poderes parecen estar aún enamorados de las estrategias que funcionaron adecuadamente durante la Guerra Fría" (2012:6). Wikileaks ha puesto de manifiesto la dificultad de la profesión diplomática para adaptarse a las tecnologías de la información. Hocking (2012) estima que "desde el telégrafo a la web 2.0, los medios sociales y Wikileaks, cómo la información se transmite entre los actores críticos en política internacional y la amplia comunidad de interesados ha generado una intensa introspección. La causa es las implicaciones para lo que los profesionales de la diplomacia hacen y cómo lo hacen". En otras palabras, la adaptación de la profesión a la transformación digital pasa por más movilidad, más capacidad de adaptación y una fuerte actividad comunicativa, principios de la diplomacia digital como hemos señalado anteriormente.

Los países han seguido diferentes métodos para adaptarse a la diplomacia digital. Francia, Suecia, Estados Unidos y el Servicio Europeo de Acción Exterior (EEAS, por sus iniciales en inglés) han emprendido acciones concretas, que muestran la 
diversidad de herramientas y planteamientos que operan en la política exterior. No existe un manual de buenas prácticas ni un criterio unificado. La práctica está sin teorizar aún, por lo que hemos preferido enumerar una acción por país.

Francia (Diplomatie.gouv,2014) ha lanzado una campaña de digitalización de su actividad, con especial atención a las redes sociales. La cuenta de Twitter@francediplo está activa desde primavera de 2009 y realiza un sesión mensual de preguntas y respuestas con el equipo del Ministerio (\#QRdiplo). Está presente en la mayoría de las redes sociales con una actividad estandarizada. En los últimos meses, ha incrementado la monitorización de las redes sociales de la mano de Agence France-Presse. La herramienta "The E-diplomacy Hub" (http://ediplomacy.afp.com) reúne información sobre las actividad de diferentes cuentas de Twitter sobre un mapa con actividad en tiempo real.

Suecia ha posicionado su diplomacia digital en torno a la Stockholm Initiative on Digital Diplomacy. Consiste en el conjunto de acciones alrededor de la diplomacia en el ámbito digital. Uno de los objetivos recogido en el plan de política exterior es liderar el proceso de transformación digital. Cuenta con el apoyo y el empeño del propio Ministro de Asuntos Exteriores, Carl Bildt, que es un reconocido tuitero y bloguero. La actividad más relevante de Stockholm Initiative on Digital Diplomacy ha sido la organización de un encuentro internacional de alto nivel con profesionales de la diplomacia, responsables de estrategia digital, académicos, activistas de los derechos humanos, periodistas y políticos de alto nivel.

Estados Unidos ha lanzado decenas de iniciativas. Cull ha explorado el largo camino de la digitalización de procesos, operaciones, recursos y prácticas en la diplomacia estadounidenses (2013). La última práctica, no incluida en su exhaustivo análisis, es la utilización de los cursos abiertos y masivos online (MOOC) para la promoción de sus intereses en materia de política exterior. El área de Educación y Cultura del Departamento de Estado ha promovido los cursos en más de 40 países con una temática que se vincula con los propios objetivos de la política exterior. En concreto, se proponen cursos de ciencia y tecnología, emprendimiento, inglés como lengua extranjera, entre otros. Tara Sonenshine, subsecretaria de Diplomacia Pública, explica que la diplomacia digital sirve a los intereses de política exterior porque los MOOC promueven la interacción con un público global, abre las puertas a la economía de la globalización, fomenta el emprendimiento y apuesta por la innovación (2013).

La EEAS orienta su diplomacia digital hacia el área de seguridad. La diplomacia digital no se caracteriza por el uso de la web social de forma sistemática, sino por su empleo sencillo, unidireccional. No está disponible en la web un manual de uso o bien otra documentación que establezca las guías de presencia en redes sociales, por lo que entendemos que existe cierta discrecionalidad en función del tema a tratar y la red. En el mismo sentido, la ausencia de unos criterios definidos de medición y rendición de cuentas dificulta la valoración de los resultados. 
El estado actual de la presencia en redes sociales es el siguiente (tabla 2)

Tabla 2

\begin{tabular}{|l|l|}
\hline Facebook & 61 perfiles \\
\hline Twitter & 36 cuentas \\
\hline Flickr & 9 perfiles \\
\hline Weibo & 1 perfil \\
\hline Tencent Weibo & 1 perfil \\
\hline Vimeo & 2 cuentas \\
\hline Youtube & 13 cuentas \\
\hline VK.com & 3 cuentas \\
\hline Storify & 1 perfil \\
\hline
\end{tabular}

Fuente: http://eeas.europa.eu/social_media/index_en.htm, noviembre 2013

La presencia en redes sociales es dispersa e irregular. No se incluyen los blogs oficiales o personales de los profesionales de la EEAS. Tampoco se vinculan los contenidos a Google Plus, cuya vinculación es automática para quienes son titulares de un cuenta de correo electrónico en Gmail. Tampoco aparece Instagram, la red social para compartir fotografías. Los diplomáticos y los profesionales no participan activamente en los medios sociales de la EEAS. La falta de personalización dificulta el liderazgo en redes sociales.

\section{Conclusiones}

La diplomacia digital es una de las áreas de investigación más interesantes en relaciones internacionales. Afecta al periodismo, a la participación ciudadana y a la alta política. Se han abierto nuevos retos (trilema de la diplomacia) y se han generado nuevas oportunidades. Los países están en proceso de innovar y transformar su acción exterior, pero aún no existe un corpus sólido de buenas prácticas. Se observan tres conclusiones.

La primera es la siguiente: la diplomacia digital está aún en unos primeros estadios. Estamos aún en una fase de aprendizaje. No se ha pensado una estrategia integral o propia, sino que se emplean las herramientas para consolidar los objetivos de la política exterior. Pocos países se atreven verdaderamente a innovar, esto es, a transformar los recursos, los procesos y los valores de la diplomacia. Francia quiere extender la francofonía. Suecia utiliza la diplomacia digital para apalancar más influencia de la que tiene. Estados Unidos invierte en distintos programas en el proceso más claro de innovación o, por precisar, de intentos de innovación. El Servicio Europeo sufre las carencias de una estrategia diplomática europea, por lo que el entorno digital apenas le reporta beneficios. 
La segunda conclusión es conservadora. La diplomacia no desaparece. Se transforma. Por eso, me resisto a aceptar que existe una "vieja diplomacia" frente a una "nueva diplomacia". La diplomacia en red es incremental y, precisamente por ello, la conectividad es un valor en alza. El acceso a la red física (Internet y nuevos medios) es solo primer en la redistribución del poder en el siglo XXI. A partir de ahí, serán los diplomáticos quienes adapten su profesión a las nuevas necesidades y demandas. La misión diplomática del futuro será aquella que integre de forma natural las tecnologías simples y concretas, adecuadas al "negocio" de la diplomacia, al tiempo que aprenda a navegar en la incertidumbre de la red.

Por último, la diplomacia digital se aventura como una idea, un desiderátum de transformación digital. La diplomacia se hace pública, esto es, se convierte en un elemento de la conversación y la lógica ciudadana. Como propuesta de valor, la diplomacia digital es una oportunidad para la participación política, el activismo y la agenda pública de intereses.

\section{Referencias bibliográficas}

ARMITAGE, R.L. y NYE, J.S. (2007) CSIS Commission on Smart Power. A Smarter, More Secure America. Washington : Center for Strategic \& International Studies

AZPÍROZ, M.L. (2013). "Framing as a tool for mediatic diplomacy analysis: study of George W. Bush's political discourse in the "War on Terror". En Communication and Society/Comunicación y Sociedad, vol. XXVI (2), 176-197

BAIRD, J. (2013). "Baird on Digital Diplomacy: Google Big Tent". Disponible en http://johnbaird.com/2013/05/30/baird-on-digital-diplomacy-google-big-tent/ [Consulta: 4/3/2014]

BECKETT, C., BALL, J. (2012). WikiLeaks: News in the Networked Era. London: Polity Books

CASTELLS, M. (2008). “Comunicación, poder y contrapoder en la sociedad red (I). Los medios y la política". En TELOS: Cuadernos de comunicación e innovación, 74, p.13-24

CASTELLS, M. (2009). Communication Power. Oxford : Oxford University Press

CLINTON, H. (2011). "Internet Rights and Wrongs: Choices and Challenges in a Networked World". Disponible en http://blogs.state.gov/stories/2011/02/15/internet-rights-and-wrongs-choices-and-challenges-networked-world\#sthash.4ImCv2o3.dpuf [Consulta: 4/3/2014]

CULL, N. J. (2013) "The Long Road to Public Diplomacy 2.0: The Internet in US Public Diplomacy". En International Studies Review, 15, pp.123-139.

CULL, N.J. (2008). "Public Diplomacy: Taxonomies and Histories". En The ANNALS of the American Academy of Political and Social Science, 616(1) pp. 31-54

ESTRELLA, R. (2011). “Diplomacia abierta, nueva diplomacia". Disponible en http:// rafaelestrella.es/2011/07/28/diplomacia-abierta-nueva-diplomacia/ [Consulta: $24 / 6 / 2014]$ 
GSTÖHL, S. (2012). "Diplomacy in the 21st Century Is Network Diplomacy". En Crossroads, III (2), p. 49-58.

GUICHOT, E. (2012). "El proyecto de ley de transparencia y acceso a la información pública y el margen de actuación de las comunidades autónomas". En Revista Andaluza de Administración Pública, 84, p. 89-134

HEINE, J. (2008) "On the Manner of Practising the New Diplomacy". En COOPER A.F., HOCKING, B. y MALEY, W., (eds.), Global Governance and Diplomacy: Worlds Apart?. Baskingstoke: Palgrave Macmillan, p. 271-287

HOCKING, B. (2006). "Multistakeholder Diplomacy: Forms, Functions, and Frustrations". En KURBALIJA, J. y KATRANDJIEV, V. (eds.), Multistakeholder Diplomacy: Challenges and Opportunities, Malta y Ginebra : DiploFoundation, p. 13-29

HOCKING, B. (2012). "(Mis)Leading propositions about 21 st century diplomacy". En Crossroads, III (2), p. 71-85.

KING, G. (2014). “The NSA Puts Journalists Under a Cloud of Suspicion”. [en línea]. CPJ Committee to Protect Journalists. Disponible en https://cpj.org/2014/02/ attacks-on-the-press-surveillance-storage.php [Consulta: 24/6/2014]

LIVINGSTON, S. (1997): "Claryfying the CNN Effect: An Examination of Media Effects According to Type of Military Intervention". Joan Shorenstein Center on the Press, Politics and Public Policy, John F. Kennedy School of Government, Harvard University. Disponible en http://www.genocide-watch.org/images/ 1997ClarifyingtheCNNEffect-Livingston.pdf [Consulta: 24/6/2014]

MANFREDI SÁNCHEZ, J.L (2011). "Hacia una teoría comunicativa de la diplomacia pública". En Communication and Society/Comunicación y Sociedad, vol. XXIV (2), 150-166

NYE, J.S. (2013). "21st Century Diplomatic Toolbox Soft Power, Economic Statecraft and Technology" [en línea]. Aspen Festival 2013. Disponible en http:// www.aspenideas.org/session/21st-century-diplomatic-toolbox-soft-power-economic-statecraft-and-technology [Consulta: 4/3/2014]

RUBIO, Rafael (2012). "El poder internacional en la sociedad en red". En RUBIO, R.; MANFREDI SÁNCHEZ, J.L.; ALONSO, G. (eds.) Retos de nuestra acción exterior: diplomacia pública y Marca España. Madrid : Ministerio de Asuntos Exteriores y Cooperación, p.19-31.

RODRIK, D. (2011). The Globalization Paradox: Democracy and the Future of the World Economy. New York : W.W. Norton \& Company

SEIB, P. (2012) Real-time Diplomacy. Politics and Power in The Social Media Era. New York : Palgrave MacMillan

SLAUGHTER, A-M. (2009): "America's Edge. Power in the Networked Century". En Foreign Affairs, p.94-113

SONENSHINE, T. (2013). "Bottom Line Diplomacy Why Public Diplomacy Matters". Disponible en http://www.state.gov/r/remarks/2013/210771.htm [Consulta: 24/6/2014] 
STAVRIDIS, J. (2013). "Disruptive Force? Or Force Multiplier?: Foreign Policy and Diplomacy in the Digital Age". Disponible en http://sites.tufts.edu/fletcherdean/ foreign-policy-and-diplomacy-in-the-digital-age [Consulta: 24/6/2014]

\section{El autor}

Juan Luis Manfredi Sánchez es profesor contratado doctor de Periodismo de la Universidad de Castilla-La Mancha. Es el coeditor de "Retos de nuestra acción exterior: Diplomacia Pública y Marca España", publicado por el Ministerio de Asuntos Exteriores y la Escuela Diplomática, y otros artículos y cursos sobre la transformación de la diplomacia.

Ha sido el responsable español del proyecto europeo MEDIADEM(European Media Policies Revisited: Valuing \& Reclaiming Free and Independent Media in Contemporary Democratic Systems), dentro del Séptimo Programa Marco (FP7-SSH-2009-A Grant agreement 244365). Ahora dirige el proyecto I+D+i CSO2013-46997-R, sobre comunicación, transparencia y participación.

En Twitter: @juanmanfredi 